\title{
Singularities in a Relativistic Pulsar Wind
}

\author{
Jianke Li \\ ANU Astrophysical Theory Centre, Department of Mathematics, \\ Faculty of Science \& the Mount Stromlo and Siding Spring Observatories, \\ Australian National University, ACT 0200, Australia \\ Received 1998 January 27, accepted 1998 July 1
}

\begin{abstract}
The nature of a singularity in a cool, gravitation-free axisymmetrical, relativistic, steady pulsar wind is further investigated, in line with the recent counter-argument of Ardavan (1995, hereafter A95) that the pure Alfvén 'singularity' has an equal importance to the Alfvén singularity if one defines the singularity via a quadratic form rather than using the ratio as adopted by Li \& Melrose (1994). The pure Alfvénic point in A95 coincides with the proposed characteristic point through which a continuous wind will meet the star and infinity. However, we find that the critical point as implied in the quadratic form is in fact the intermediate point (Ardavan 1979), but not the pure Alfvénic point. Thus the analysis of A95 does not indicate any significance of the pure Alfvénic point. We also demonstrate that the intermediate point which appears in the quadratic form of A95 is not genuine.
\end{abstract}

Keywords: Magnetohydrodynamics — pulsars: general — stars: magnetic fields

\section{Introduction}

The discovery of rapidly rotating neutron stars with a magnetic field $\sim 10^{12} \mathrm{G}$ or $10^{8} \mathrm{~T}$ in the late 60 s changed our view about stellar degenerate matter, and revealed the existence of an astrophysical laboratory for electrodynamics. A rapidly rotating conducting neutron star with a strong magnetic field will induce significant electric fields across the magnetic field lines. For a typical rotation rate (e.g. 0.03 s for the Crab pulsar) and the typical magnetic field strength shown above, the electric field above the stellar surface $\left(\sim 10^{13} \mathrm{~V} \mathrm{~m}^{-1}\right)$ has a component along a field line and is strong enough to pull the charged particles out of the stellar surface to form a pulsar magnetosphere, despite the huge surface gravity (Goldreich \& Julian 1969; Mestel 1971). Such a magnetosphere is responsible for generating spin-modulated radio emission which led to the discovery of these pulsing neutron starspulsars. The strong centrifugal effect, due to the rapid stellar spin, becomes dominant at large distances, and a pulsar wind inevitably develops (cf. e.g. Mestel \& Shibata 1994; Michel 1969; Goldreich \& Julian 1970). Understanding these magnetospheres and associated pulsar winds has been one of the important frontiers of theoretical research.

In comparison with an outflow from a main sequence star, the pulsar wind is different as the special relativity effect plays an important role. The pressure and even the gravitational forces acting on the wind plasma, however, may not be as important as the electromagnetic and centrifugal forces. Theoretical modelling of the wind thus often neglects thermal and gravity effects, and such a wind is usually called a gravity-free, cold relativistic wind (see Michel 1969). An analytic approach usually assumes mass conservation and an ideal plasma (dissipation-free). The former is probably the crudest one of all, as the plasma may be generated from electron-positron pair production along curved field lines in the the magnetosphere (e.g. Sturrock 1971; Ruderman \& Sutherland 1975), indicating that the inner boundary conditions for a wind may not be well-defined. But nevertheless, one may ignore this complication and assume that ideal MHD equations are applicable to the whole domain. Assuming a steady state and axisymmetry, the ideal MHD equations for a pulsar wind can be integrated along a poloidal field line. Although the transfield equation (e.g. Okamoto 1975; Okamoto 1978, hereafter O78; Ardavan 1979, hereafter A79) cannot be simply integrated (when considering the three dimensional magnetic field structure), a solution for a cold, gravity-free relativistic pulsar wind derived only along a poloidal field line, or the 'standard solution', is of particular theoretical importance, as it is the simplest and has perhaps inherited the most important physics for a realistic pulsar wind.

The singular nature is one of the important issues for a standard wind. The wind solutions, which relate wind quantities to integral constants, are singular for a physical quantity, and some work (see below) even indicates the 'multi-singular' nature. It is therefore an important matter whether a singular feature is physical or unphysical. The alternative way to phrase the question is whether the singularity is significant or not. We say that a singularity is significant when it physically constrains 
the flow parameters, and we say it is spurious when it may only arise from a special mathematical form, without constraining the flows. O78 found two mathematical singularities associated with the relativistic 'splitting' of the conventional Alfvénic point, i.e. the Alfvénic and the pure-Alfvénic points. O78 argued that the Alfvénic point may be more significant than the pure-Alfvénic point, though the later singularity might still have physical significance. It was suggested by (A79) that, in addition to the previous two important singularities, there exists another equally important singularity, the intermediate point, though $\mathrm{O} 78$ did not argue for the existence of the intermediate one. The analysis of A79 involves a selection of different variables other than the Lorentz factor $\gamma$, leading to different 'singularities'. However, other derivations (e.g. Michel 1969; Goldreich \& Julian 1970; Kennel, Fujimura \& Okamoto 1983; Camenzind 1986) do not indicate the existence of the pure Alfvénic point and also the intermediate one. Thus some confusion and inconclusiveness remain in the literature about the singular nature of the standard pulsar wind. The complication is entirely due to special relativistic effects, because in the classic stellar wind domain all the proposed three singularities merge into one, the Alfvénic point. Resolving the multi-singular nature of a pulsar wind is clearly not trivial, as this would answer the question whether the special relativistic effects impose more constraints on the wind flow than for the nonrelativistic case. Li \& Melrose (1994, thereafter LM94) studied the problem and have shown that only the Alfvénic point is genuine, and once the equations are regularised at the Alfvénic point, other singularities disappear and therefore they are not genuine. The argument is consistent with the physical nature - wave-singularity analogy (see LM94). The unequivocal implication is that the relativistic effect does not yield additional constraints on the wind solution.

However, in a recent paper, Ardavan (1995; A95) reiterated his early argument (A79), and therefore criticised LM94. A95 emphasised that a singularity does not necessarily arise from a ratio of variables as defined at a point in which the denominator goes to zero (and so the numerator must also go to zero). A95, following A79, used a quadratic form of certain variables and discussed the determinant in relation to the two branch solutions. A79 and A95 argued that different singularities might arise by choosing different variables, and these singularities all have the same physical significance. A95 concluded that LM94 considered only a part of the linearised version of singular analysis, and the definition of a singularity could be more general, for instance with the use of a quadratic form.

In fact, the conclusion of A95 is the fundamental argument which we believe may have caused confusion in the literature, since it emphasises the importance of a variation of mathematical form rather than in physical nature, i.e. whether it helps to constrain the flow or not. In this paper we attempt to clarify this matter. We first summarise and study the argument of A95 in Section 2, where we show that the characteristic point which A95 argued is the intermediate point but not the pure Alfvénic point, and then demonstrate in Section 3 that the intermediate point, is not genuine, and finally conclude in Section 4. (We use the symbols adopted in A95 simply for an easy comparison.)

\section{Characteristic Point}

The basic integrals along a magnetic field line for the standard pulsar wind are (O78; A79)

$$
\begin{gathered}
v_{\phi}=\kappa B_{\phi}+r \omega, \\
\kappa \rho=\left(v_{p} / B_{p}\right) \rho=F \\
-r B_{\phi} /(4 \pi)+r \gamma \rho v_{\phi} \kappa=G, \\
\gamma\left(1-\frac{r \omega}{c} \frac{v_{\phi}}{c}\right)=\exp H
\end{gathered}
$$

In the above formulae subscript $p$ and $\phi$ denote the poloidal and toroidal components respectively, $\gamma$ is the Lorentz factor, $\omega, F, G$ and $\exp H$ are integral constants along a field (or poloidal field) line, and the other quantities have their usual meaning. Integrals (1) to (4) are in order the generalised Ferraro isorotation law (the integral of the induction equation), mass flux conservation, angular momentum conservation and finally energy conservation. Setting $\gamma=1$, these equations reduce to those derived earlier by Mestel (1968), except the difference in (4) due to neglecting the pressure and gravity terms. Most important is the Lorentz factor $\gamma$, which characterises the relativistic nature of a pulsar wind. It can be expressed by

$$
\gamma=\frac{\left(1-\hat{r}_{A}^{2}-4 \pi F \gamma \kappa\right) \exp H}{\left(1-\hat{r}_{A}^{2}\right)\left(1-\hat{r}^{2}-4 \pi F \gamma \kappa\right)},
$$

where

$$
\hat{r}_{A}=\left(1+c^{2} F \exp H / \omega G\right)^{-1 / 2}, \quad \hat{r}=r \omega / c .
$$

It can been seen that there is a unique singularity at $\hat{r}=\hat{r}_{A}$ in (5), though the regularisation at the Alfvénic point has been made. Note that (5) is a complete expression as the RHS has no dependence on $\gamma(\gamma \kappa$ is independent of $\gamma)$. The quantity $\gamma$ is thus a function of integral constants, the proper 
density and also the distance. The toroidal velocity $v_{\phi}$ may be expressed as

$$
v_{\phi}=\frac{r \omega-4 \pi G \kappa / r}{1-4 \pi F \gamma \kappa} .
$$

Note that (7) is not a complete form because the numerator is a function of $\gamma$ which is subject to its singular nature. It turns out that once $\gamma$ is regularised at $\hat{r}_{A}$, i.e. equation (5), $v_{\phi}$ in (7) is automatically singular free at the pure Alfvénic point (see LM94). Nevertheless, once

$$
4 \pi F \gamma \kappa=1
$$

which is the definition of the so-called pure Alfvénic point, we must require

$$
r \omega=4 \pi G \kappa / r
$$

One can in principle express (5) in a quadratic form as adopted in A95:

$$
\Gamma^{2}-\left[\left(1-\hat{r}^{2}\right) R+\left(1-\hat{r}_{A}^{2}\right)^{-1}\right] \Gamma+R=0,
$$

where the new variables are

$$
\Gamma=e^{-H} \gamma, \quad R=\left(4 \pi F^{2} e^{H}\right)^{-1} \rho .
$$

We bear in mind that (10) is not a complete quadratic expression as $\Gamma$ and $R$ are not independent. However, this does not affect having two solutions of $\Gamma$ :

$$
\begin{aligned}
\Gamma^{ \pm}= & \frac{1}{2}\left[\left(1-\hat{r}^{2}\right) R+\left(1-\hat{r}_{A}^{2}\right)^{-1}\right] \\
& \pm\left\{\frac{1}{4}\left[\left(1-\hat{r}^{2}\right) R+\left(1-\hat{r}_{A}^{2}\right)^{-1}\right]^{2}-R\right\}^{1 / 2} .
\end{aligned}
$$

A95 discussed the two solutions and found that $\Gamma^{+}$and $\Gamma^{-}$behave well at infinity and within $\hat{r}_{A}$ respectively. Thus the continuity of a smooth solution requires a transition from $\Gamma^{-}$to $\Gamma^{+}$(outwards) at a point $\Gamma^{+}=\Gamma^{-}$. This point has been shown by A95 to occur at $\hat{r}>\hat{r}_{A}$ and we may call it a characteristic point. Two extra conditions from (12) for the characteristic point are readily obtained:

$$
\begin{gathered}
\Delta=\left[\left(1-\hat{r}^{2}\right) R+\left(1-\hat{r}_{A}^{2}\right)^{-1}\right]^{2}-4 R=0, \\
\Gamma=\frac{1}{2}\left[\left(1-\hat{r}^{2}\right) R+\left(1-\hat{r}_{A}^{2}\right)^{-1}\right] .
\end{gathered}
$$

Alternatively, (13) and (14) define the characteristic point. This leads to

$$
\Gamma=\sqrt{R}
$$

It is important to realise that A95 does not give the correct form (15) but $\Gamma=R$ instead. By (15), we obtain an equivalent relation

$$
\left(4 \pi F \gamma^{2} \kappa\right)_{c}=\exp H
$$

With the use of (4), (16) becomes

$$
1-(4 \pi F \gamma \kappa)_{c}-\left(\frac{r \omega}{c} \frac{v_{\phi}}{c}\right)_{c}=0 .
$$

According to A79, (17) defines the intermediate point.

For the pure Alfvénic point, one can derive a different relation as compared to (15). Expressing (11) as

$$
R=\Gamma / 4 \pi F^{2}(\gamma / \rho),
$$

we find that the pure Alfvénic point, as defined by (8), corresponds to

$$
\Gamma=R
$$

The difference between (19) and (15) shows that the intermediate point is independent of the pure Alfvénic point. We thus conclude that the quadratic analysis of A95 has no relevance to the pure Alfvénic point.

\section{Is the Intermediate Point Genuine?}

The quadratic form allows one to incorporate some arguments about the boundary conditions both near the stellar surface and infinity, and as a result the special point $\Gamma^{-}=\Gamma^{+}$looks like 'important' as it yields an extra condition (15). By analogy with nozzle-type flows in aerodynamics, where critical points exist, we see that a subsonic flow for the inner boundary and a supersonic flow for the outer boundary are satisfied simultaneously simply by having a transonic flow through the critical point. Our question is whether the intermediate point resembles such a point or not?

As we have already seen in Section 2, (5) is a regularised expression for the Lorentz factor $\gamma$, and in contrast, the quadratic form (10) is incomplete in that both $\Gamma$ and $R$ are coupled with $\gamma$. The quadratic analysis is thus based on having coefficients (of quadratic expression) which are not independent of the variable $\Gamma$. To realise this is important because a characteristic point in such a situation is not unique and different characteristic points can be generated.

We may write (10) in a different form:

$$
(1+k) \Gamma^{2}-\left[\left(1-\hat{r}^{2}\right) R+\left(1-\hat{r}_{A}^{2}\right)^{-1}-k \Gamma\right] \Gamma+R=0,
$$


where $k$ is an arbitrary constant. Because (20) is identical to (10), following A95 we still argue that $\Gamma^{-}$must connect to $\Gamma^{+}$at a characteristic point. The solutions are

$$
\begin{aligned}
\Gamma^{ \pm}= & \frac{1}{2(1+k)}\left[\left(1-\hat{r}^{2}\right) R+\left(1-\hat{r}_{A}^{2}\right)^{-1}-k \Gamma\right] \\
& \pm \frac{1}{2(1+k)}\left\{\left[\left(1-\hat{r}^{2}\right) R+\left(1-\hat{r}_{A}^{2}\right)^{-1}-k \Gamma\right]^{2}\right. \\
& -4(1+k) R\}^{\frac{1}{2}}
\end{aligned}
$$

To require $\Gamma=\Gamma^{-}=\Gamma^{+}$at the characteristic point, we obtain two conditions:

$\left[\left(1-\hat{r}^{2}\right) R+\left(1-\hat{r}_{A}^{2}\right)^{-1}-k \Gamma\right]^{2}=4(1+k) R$,

$\left[\left(1-\hat{r}^{2}\right) R+\left(1-\hat{r}_{A}^{2}\right)^{-1}-k \Gamma\right]=2(1+k) \Gamma$.

Combining (20) and (21), we obtain

$$
\Gamma=(1+k)^{-1 / 2} \sqrt{R}
$$

By (11), equation (24) leads to

$$
4 \pi F \gamma^{2} \kappa=\frac{1}{(1+k)} \exp H
$$

With the use of (4) again, (25) becomes

$$
1-4 \pi F \gamma \kappa-\left(\frac{r \omega}{c} \frac{v_{\phi}}{c}\right)-k(4 \pi F \gamma \kappa)=0
$$

Clearly for $k \neq 0, \quad(26)$ describes a point other than the intermediate one, and there can be many different points for arbitrary $k$. Because they are created by having a non-zero $k$ for the same relation (10), they are spurious and therefore have no significance. The ease of generating all these spurious points stems from the dependence between the coefficients and $\Gamma$ of the quadratic form, in a similar manner to dependent variables on both sides of a ratio (see LM94). Since the intermediate point is derived on the condition that $\Gamma$ and $R$ are dependent, we argue that it is created rather than being a genuine one. Once $\Gamma$ is expressed by $\gamma$ as an independent variable, i.e. equation (5), the intermediate point disappears.

\section{Conclusion}

The characteristic point which arises from the quadratic analysis of A95 is the intermediate point in A79, but not the pure Alfvénic point as argued in A95. Thus the quadratic analysis in A95 does not show any significance of the pure Alfvénic point. We also demonstrate that a quadratic analysis may generate non-genuine characteristic points, and the intermediate point is one of them.

\section{Acknowledgments}

The author thanks Martijn deKool for reading the manuscript.

\section{References}

Ardavan, H. 1979, MNRAS, 189, 397

Ardavan, H. 1995, MNRAS, 273, 1129

Camenzind, M. 1986, A\&A, 162, 32

Goldreich, P., \& Julian, W. H. 1969, ApJ, 157, 869

Goldreich, P., \& Julian, W. H. 1970, ApJ, 160, 971

Kennel, C. F., Fujimura, F. S., \& Okamoto, I. 1983, J. Astrophys. Geophys. Fluid Dyn., 26, 147

Li, J., \& Melrose, D. B. 1994, MNRAS, 270, 721

Mestel, L. 1968, MNRAS, 138, 359

Mestel, L. 1971, Nature, 233, 149

Mestel, L., \& Shibata, S. 1994, MNRAS, 271, 621

Michel, F. C. 1969, ApJ, 158, 727

Okamoto, I. 1975, MNRAS, 173, 357

Okamoto, I. 1978, MNRAS, 185, 69

Ruderman, M. A., \& Sutherland, P. G. 1975, ApJ, 196, 51 Sturrock, P. A. 1971, ApJ, 164, 529 